\title{
A computer system to assist in the evaluation of the EEGs of epileptic patients
}

\author{
J. GOTMAN \\ Montreal Neurological Institute and Department of Neurology and Neurosurgery, McGill University \\ Montreal, Quebec H3A 2B4, Canada
}

\begin{abstract}
The specific EEG manifestations of epilepsy, seizures, and interictal spikes and sharp waves occur at unpredictable times and at variable frequeacies. To obtain an adequate diagnosis, it is often necessary to record the EEG for several hours or several days. A computer system was developed to perform data reduction and quantification by continuously monitoring seizures and automatically recognizing interictal spikes and sharp waves. The pest 2 min of EEG are kept on the computer disk at every instant. When an epileptic seizure occurs or when a spike is detected, a sample of EEG, including a section preceding the event itself, is written on the EEG polygraph and on magnetic tape. A continuous recording is thus replaced by samples of varying lengths, containing only the important aspects of the EEG, reducing considerably the original data. After the monitoring session, the spatial and temporal distributions of the interictal activity are presented in a quantified form on the terminal. The seizures are recorded on digital tape and are available for several types of processing. The patient is also monitored by a video system; EEG and video are synchronized by a time-of-day clock to allow electroclinical correlations.
\end{abstract}

Computers have been used for a long time to process various aspects of the electroencephalogram (EEG). Except for the work related to the automatic scoring of sleep stages, computer processing has been mostly aimed at analyzing in a quantitative fashion sections of EEG varying in duration from a few seconds to a few minutes. The results obtained using techniques such as spectral analysis or time-domain analysis consist of a quantitative and simplified description of some aspects of the original EEG section. There are several reviews of these methods and the results obtained with them (Barlow, 1979; Cox, Nolle, \& Arthur, 1972; Gevins, Yeager, Diamond, Spire, Zeitlin, \& Gevins, 1975). At the Montreal Neurological Institute, we have concentrated on characterizing the EEG in patients having supratentorial space occupying lesions (Gotman, Gloor, \& Ray, 1975; Gotman, Skuce, Thompson, Gloor, Ives, \& Ray, 1973) and the interictal manifestations of epilepsy (Gotman \& Gloor, 1976; Gotman, Gloor, \& Schaul, 1978).

Before describing our computer system for the longterm monitoring of epileptic patients, I would like to review briefly the most prominent EEG manifestations of epilepsy and why it is necessary to record them. Different types of epilepsy have different EEG manifestations, and knowledge of the type of epilepsy is important to adjust medical treatment. In some patients, the drug treatment may prove ineffective and a surgical removal of the epileptic focus may be considered. In

This work was supported in part by Operating Grant MA-7204 from the Medical Research Council of Canada. The author is supported by a scholarship from the Medical Research Council of Canada. these cases, it is extremely important to determine the area of the brain that is responsible for abnormal electrical discharges. The recording of the EEG from multiple scalp locations plays a critical role in this determination.

EEG abnormalities specific to epilepsy are of two types: (1) Ictal activity is present during clinical epileptic seizures; it often starts with a rhythmic discharge varying in frequency from 2 to $25 \mathrm{~Hz}$ or with a "flattening" of the record (Figure 1). During the seizure, the amplitude usually increases and numerous types of patterns may be present, and electromyographic (EMG) activity from contracting scalp muscles is often mixed with the EEG during seizures. (2) Interictal activity is present between seizures and has no concomitant clinical manifestation; it consists of spikes and sharp waves (Figure 2). Both ictal and interictal activity occur at random. Seizures may occur at frequencies ranging from several times a day to once a month, and spikes and sharp waves may occur at frequencies ranging from every few seconds to every few hours.

The traditional way of recording the EEG in epileptic patients is to use 8 or $16 \mathrm{scalp}$ locations over $30 \mathrm{~min}$ to a few hours; most often, spikes and sharp waves are obtained, and sometimes a seizure may also be recorded. The system described here attempts to improve efficiency by allowing longer recordings and by storing important patterns in the computer for further processing.

\section{OVERVIEW OF THE SYSTEM}

The present set-up is based on an earlier system described by Ives, Thompson, and Gloor (1976). Expensive, long, and difficult to interpret records are assumed 
$-2-48-$

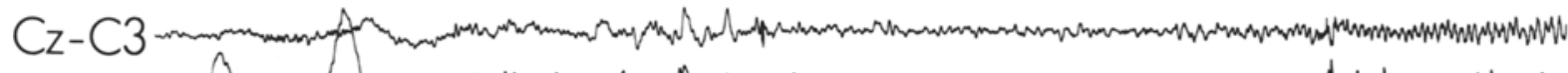

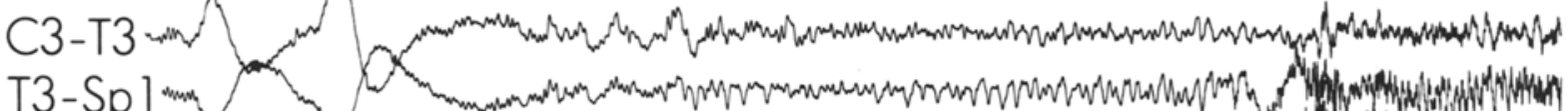

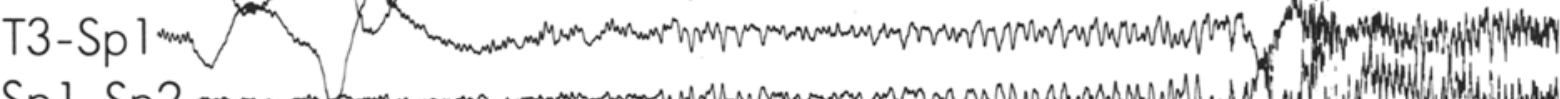

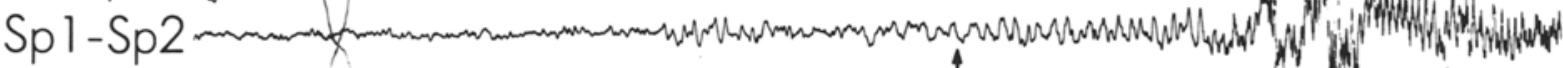
Sp2-T4

$\mathrm{T} 4-\mathrm{C} 4$ $\mathrm{C} 4-\mathrm{Cz}$ C. 2 SEC $\int 100 \mu \mathrm{V}$

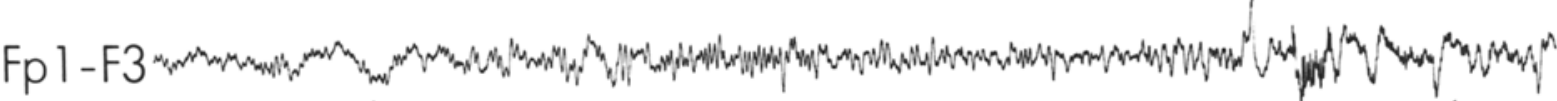
F3-C3 - 3 C3-P3 P3-O 1 Fp2-F4 F4-C4 C4-P4 P4-O2

Figure 1. Bipolar recording of the onset of an epileptic seizure. Electrode names correspond to the International 10-20 system (Sp1 and Sp2 are left and right sphenoidal electrodes). The onset of the seizure is marked by an arrow. Note the time of day written by the EEG machine at the top and the letters "P-B," indicating that this section of EEG was recorded because the seizure pushbutton was pressed.

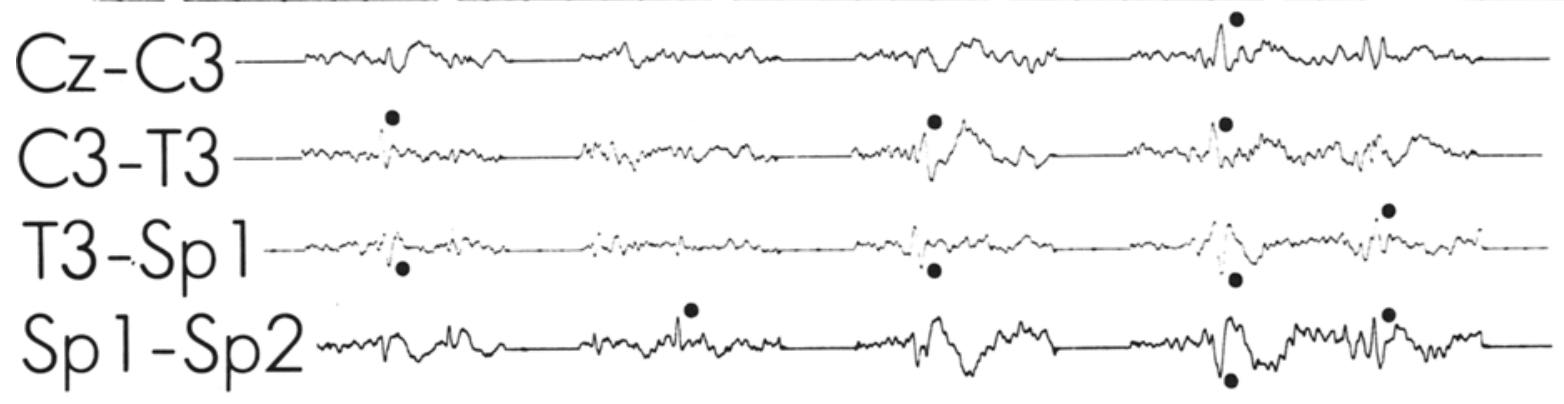

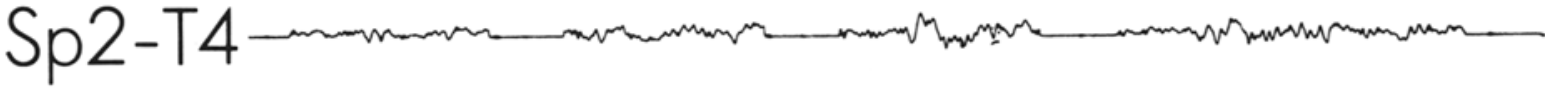

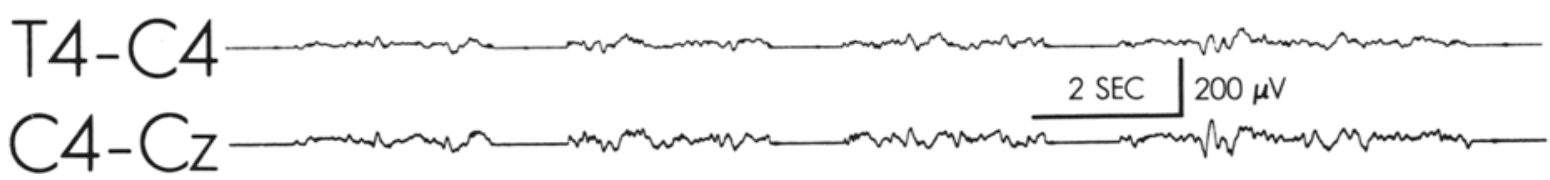

Figure 2. Sections of EEG with automatically detected spikes and sharp waves marked with a dot. Actual time between these sections is not known. Note " $S$ " at the top indicating that these sections of EEG were recorded because of a spike detection by the program. 
to contain mostly unnecessary information and are replaced by a discontinuous and much shorter recording, including only those sections of the EEG that are likely to be of interest for epileptic patients. The choice of a section to be recorded can be made on the basis of behavioral manifestations (the patient is having a seizure) or because the computer program has detected an interesting EEG pattern. It is often important, however, to obtain the EEG preceding the event that triggered the recording. This is the case for epileptic seizures, in which it is most important to record the onset, but clinical signs of the seizure may not become apparent until 10 or $20 \mathrm{sec}$ after onset. Similarly, when a program detects a spike, it is necessary to retain a section of EEG including 1 or $2 \mathrm{sec}$ prior to the spike.

During recording, the 16 channels of EEG are amplified and time-division multiplexed in to one signal in a small unit placed on the patient's head. This signal is transmitted to the computer room of the EEG laboratory and is demultiplexed, filtered $(.5$ to $70 \mathrm{~Hz})$, and made available on the 16 analog-to-digital converters. A computer program samples the EEG and writes it on disk, using the disk as a circular buffer so that, at every instant, the last 2 min of EEG are recorded (Figure 3). As the current EEG is written on disk, the program reads from the disk the EEG from the preceding 2 min and makes it available on the digital-to-analog converter. The 16 channels are also time-division multiplexed and written out in traditional form on an EEG polygraph.

Simultaneously with the EEG monitoring, the patient's behavior is recorded on a time-lapse video system (Ives \& Gloor, 1978).

\section{DETAILS OF IMPLEMENTATION}

\section{Computer System}

The system consists of a Digital Equipment Corporation PDP-11/60 computer with 64K of 16-bit-word memory and a floating-point processor. The system mass storage device is a set of two 5-megabyte RL01 disks. Auxilliary storage devices include a dual RX02 floppy

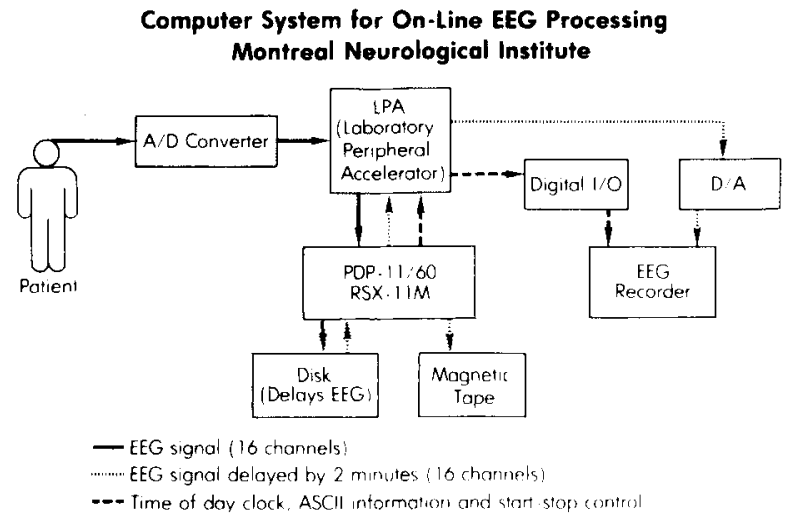

Figure 3. Organization of the computer system for EEG monitoring. disk and a TE10 800-bpi magnetic tape. The laboratory peripheral accelerator (LPA-11) is a microprocessorcontrolled device that handles analog-to-digital, digitalto-analog, and digital input/output operations through direct memory access. Thus the CPU of the computer is relieved of a large part of the constraints of real-time operation. The results are displayed on a Tektronix 4012 storage terminal with hard-copy unit. A 16-channel EEG playback unit writes the EEG coming from the computer. This unit is equipped with a character generator that can receive ASCII code. Text can be written at the top of the EEG paper, as shown in Figures 1 and 2.

Programs run under the RSX-11M operating system (Version 3.2). Except for a small number of subroutines written in MACRO 11, programs are written in FORTRAN IV PLUS. No special system software is necessary.

\section{Organization of Programs}

Two main programs run simultaneously: MONITOR performs the analog data input and output, disk, and magnetic tape operations; MONSPI performs the online processing of the incoming EEG data. The two tasks communicate through global event flags and a common data area.

MONITOR samples the 16 channels of EEG at $200 \mathrm{samples} / \mathrm{sec} / \mathrm{channel}$, using triple buffering; each buffer has 1,024 words ( 64 words/channel, hence $.320 \mathrm{sec}$ of EEG). The three buffers are in common memory because they must also be accessible by the processing program MONSPI. One buffer is processed while another one is filled with incoming data. Because of the method used, the time needed to process one buffer varies with the type of EEG waveform and may occasionally exceed the time required to fill a buffer. Triple buffering provides the necessary flexibility to operate in real-time. At the same time one buffer is processed, it is written on the disk to create the 2-min delay. The buffer written on disk 2 min earlier is read from the disk, using double buffering. While one buffer is read from disk, the other is sent on the digital-toanalog converter, to be available on the EEG machine.

MONITOR also reads the digital input word to know if the seizure pushbutton, placed next to the patient, is being pressed. It writes a digital output word that consists of 1 bit for the control of start and stop of the EEG machine and 8 bits with the ASCII code of the character to be written by the character generator of the EEG machine. In this way, the time of day is written every $10 \mathrm{sec}$ on EEG polygraph paper (Figure 1). This is useful for a joint examination of the EEG and the behavior recorded on videotape. Other characters indicate whether the EEG was started by the seizure pushbutton or the pattern recognition program.

The program MONSPI performs the automatic recognition of epileptic spikes and sharp waves. By using event flags, MONITOR indicates to MONSPI when a 
buffer is available for processing. MONSPI indicates in return to MONITOR when the EEG machine and the magnetic tape should be started and stopped following the recognition of a spike or sharp wave. MONSPI also saves on a disk file the parameters of all detected spikes necessary for an off-line processing of these events, as described below.

\section{Spike Recognition Method}

Recognition of spikes and sharp waves is described by Gotman and Gloor (1976), and its extension for prolonged recording is described by Gotman, Ives, and Gloor (1979). A thorough evaluation of the method applied to short recordings was performed by Gotman et al. (1978). The method consists of a set of steps performed on-line and a set performed off-line.

On-line operations. On-line operations include the following: (1) Prefiltering is done with a digital filter to remove all possible $60-\mathrm{Hz}$ contamination. (2) Each EEG channel is broken down into half-waves with provision for elimination of small-amplitude segments. (3) Measurement of amplitude of each half-wave is performed relative to the average amplitude of the half-waves in the preceding $5 \mathrm{sec}$. This is necessary because spikes and sharp waves are not defined in absolute amplitude, but relatively to the background of the EEG (Chatrian, Bergamini, Dondey, Klass, LennoxBuchthal, \& Petersen, 1974). The duration of each half-wave is also measured. (4) Every set of two adjacent half-waves is tested (making a wave) to see if amplitudes and durations fall within the set of limits empirically defined for spikes and sharp waves (amplitude and duration thresholds vary with each other). If the test result is positive, the second derivative at the apex of the wave measured relative to the background amplitude is also taken into consideration to decide if a wave is a possible spike or sharp wave. The second derivative at the apex measures the "sharpness," an important factor in human visual recognition. (5) A test is performed to determine the presence of movement or EMG artifact (as indicated by very large-amplitude sections of the EEG or a large number of short-duration waves). If any such artifact is detected in a channel during processing one buffer, all possible spikes and sharp waves detected in that buffer are cancelled. (6) If a spike or a sharp wave is detected, the MONITOR program is notified and the appropriate section of EEG is written on magnetic tape and on the EEG recorder. The parameters (channel number, amplitude, duration, sharpness, and a few others) of each spike and sharp wave are appended to the spike parameter file on the disk.

Off-line operations. When the monitoring session is terminated, further processing of the results of the spike and sharp wave recognition procedure may be performed. The sections of EEG written on magnetic tape may be played back on the EEG machine, as well as displayed on the Tektronix terminal. During the display on the terminal, the user may edit the results.
False detections may be removed from the spike parameter file, so that the final computer displays (Figures 4A and 4B) may reflect only valid detections. Additionally, the final computer displays may be changed to include only certain channels instead of all of them (Figure 5).
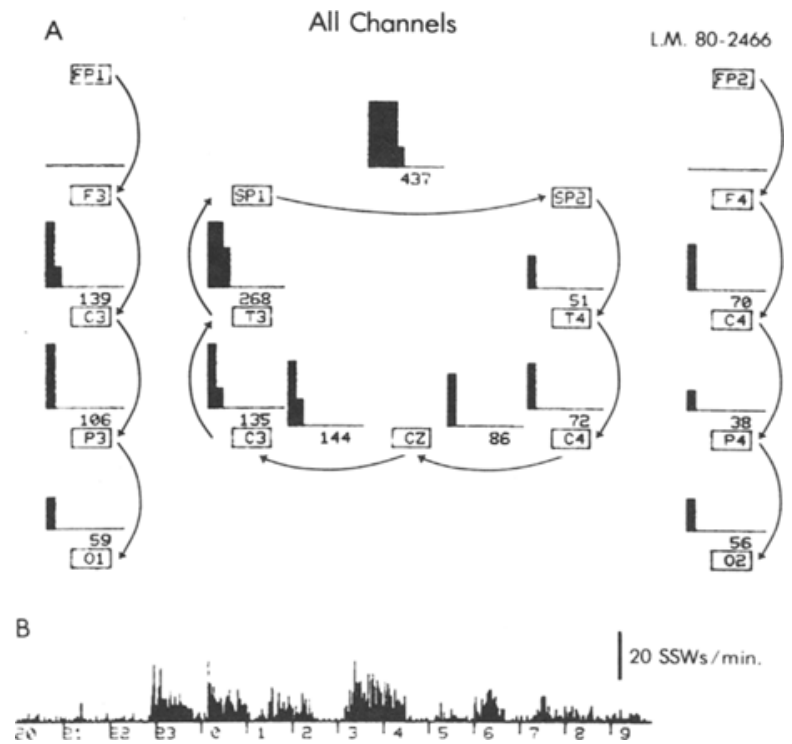

Figure 4. Results of the automatic spike recognition during a 14-h recording. (A) Spatial distribution of the spikes and sharp waves: Arrows joining electrode names indicate bipolar channels; the number of spikes or sharp waves found in each channel is indicated and illustrated by a graph. (B) Temporal distribution of spikes and sharp waves between $2000 \mathrm{~h}$ and $1000 \mathrm{~h}$ the next day, showing prominence of activity during sleep, as well as a cyclic appearance of this activity.

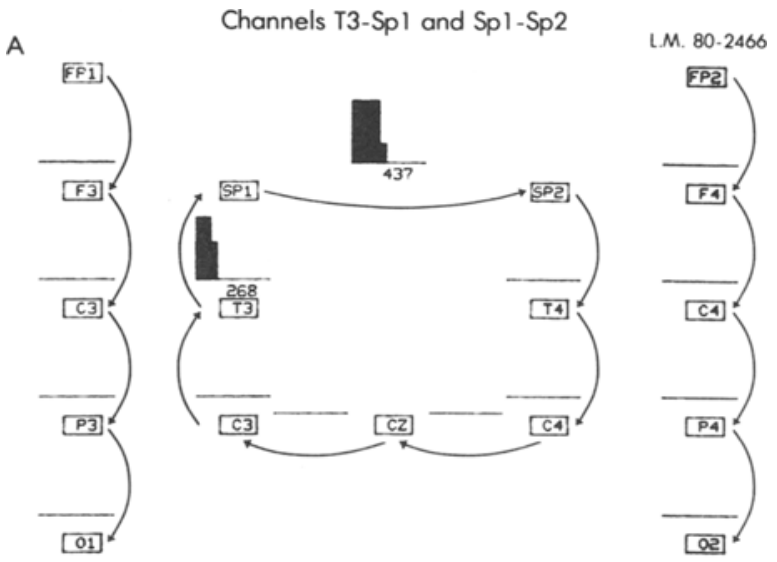

B

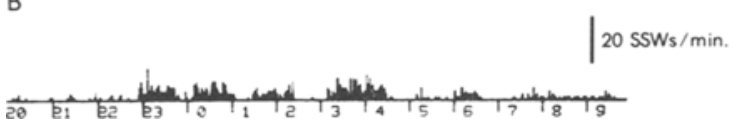

Figure 5. Subset of Figure 4, in which data from two EEG channels were extracted. (A) Spatial distribution. (B) Temporal distribution showing that the detected events in these two channels have a temporal distribution similar to that of all the channels taken simultaneously. 
A BEFORE DIGITAL FILTERING 0.3 SEC, $70 \mathrm{HZ}$ SINGLE POLE

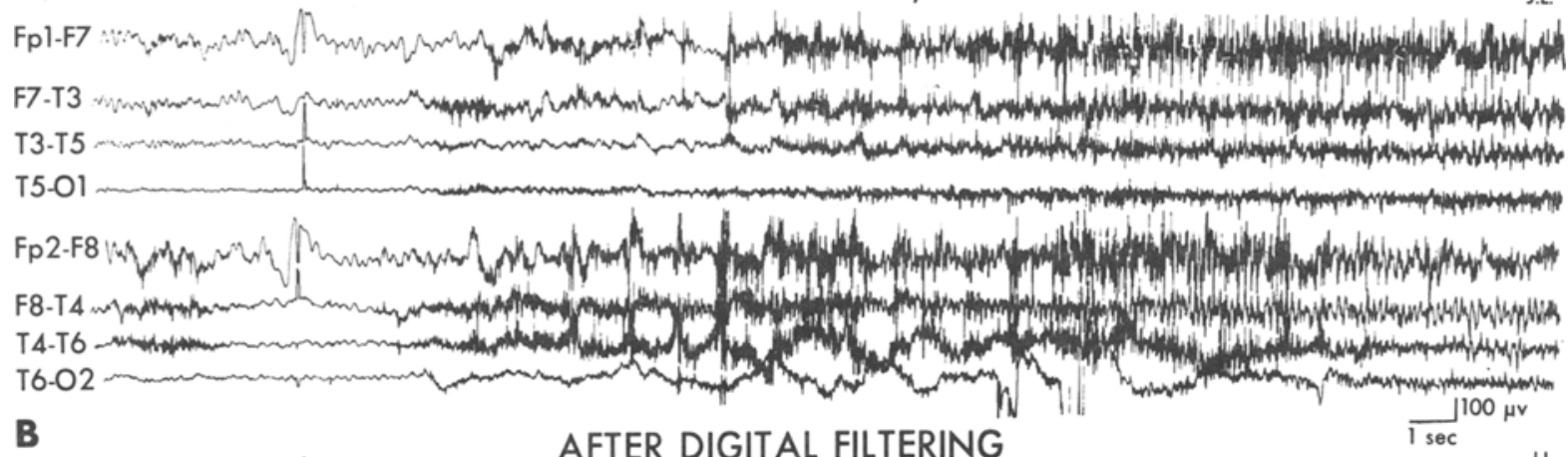

B

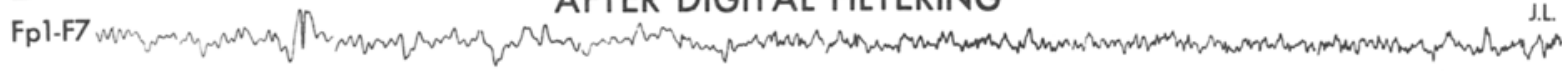

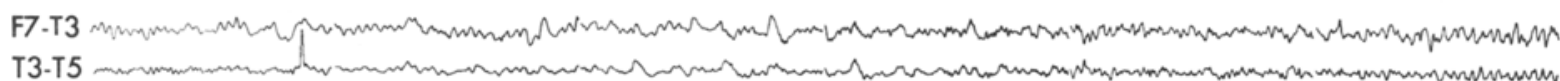

T5-01

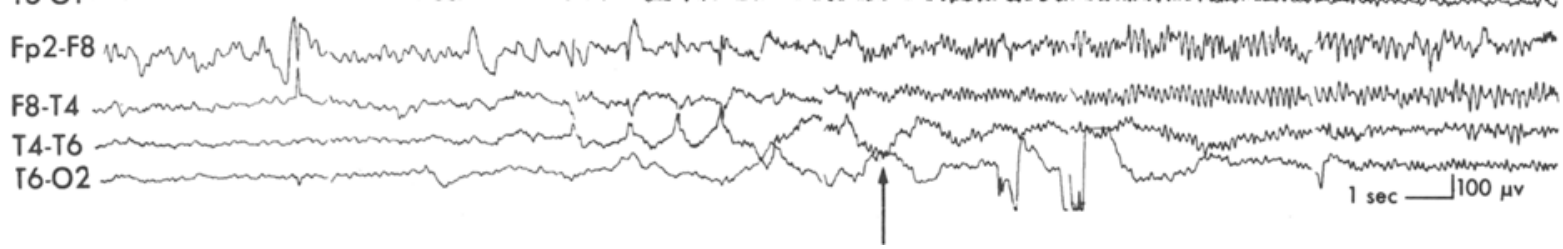

Figure 6. The problem of EMG artifact during the recording of an epileptic seizure. (A) Eight channels of EEG showing a traditional recording of the seizure onset contaminated by EMG activity. (B) Digital filtering of the signal reveals the rhythmic 8-Hz EEG activity in the right hemisphere (lower four channels; arrow shows onset).

The computer displays in Figures 4 and 5 give a concentrated and easily interpretable view of a large amount of data covering many hours of recording. They are meant to be used by an electroencephalographer, who would not necessarily be familiar with any of the details of the spike and sharp wave recognition method.

\section{Processing of Seizure Data}

The most common way of triggering the recording of epileptic seizures is through the "seizure pushbutton," which is pressed by the patient or a nurse at the time of a seizure. Some patients have seizures with minor clinical manifestations of which they may not be conscious and which may not be witnessed. An automatic seizure recognition procedure is being developed at present for such situations.

A difficulty frequently arises in the interpretation of the recording of an epileptic seizure. The electrical activity of cerebral origin is obscured by EMG activity originating in scalp muscles that contract during the seizure. An example of such a seizure is shown in Figure $6 \mathrm{~A}$. We have developed a procedure that, in some cases, allows us to remove the EMG and reveal the underlying EEG activity. The method originates from the two following observations: (1) Rhythmic activity at the beginning of seizures usually has a frequency below $25 \mathrm{~Hz}$ (Gotman, Ives, \& Gloor, Note 1), and (2) EMG activity has most of its energy above $25 \mathrm{~Hz}$ and usually does not have any rhythmic component; hence, it normally has no clear peak in its frequency spectrum. It is important to remember that this observation is derived from the analysis of voluntary contractions of muscles (O'Donnell, Berkhout, \& Adey, 1974). We assume that muscular contractions during epileptic seizures have similar characteristics. This has been our experience in many seizures, but not in all. Hence, the method is not applicable when it is considered that the EMG may have a rhythmic component below $25 \mathrm{~Hz}$.

The onset of the EMG-contaminated seizure recording is subjected to spectral analysis (Figure 7). If the spectra of some channels show a peak below $25 \mathrm{~Hz}$, then the record may be filtered with a sharp digital finite-impulse response (FIR) filter to eliminate EMG and reveal the time course of rhythmic activity of cerebral origin (Figure 6B).

Another type of off-line processing of recorded seizure consists of performing a cross-spectral analysis between different EEG channels. The measurement of phase and coherence may allow one to study the propagation of seizure activity from one cerebral location to another (Brazier, 1972).

\section{User Interaction}

The interactions with the monitoring program and with the off-line processing programs are made as simple as possible so that they can be used by EEG technologists 
Ist seizure (10 sec)

Left

\section{Right}

J.L.
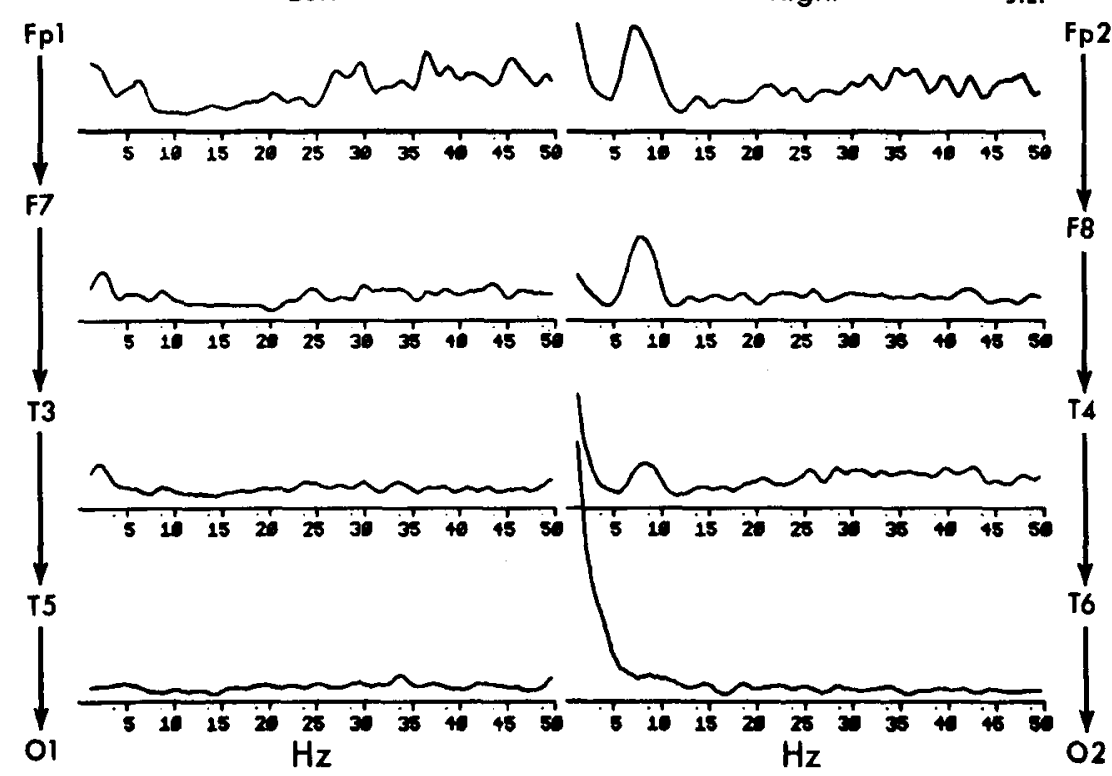

Figure 7. Frequency spectra of the seizure onset shown in Figure 6. The four channels on the left hemisphere show the typical spectra of EMG with high-energy fast activity. The upper three channels on the right show similar spectra with, additionally, a prominent peak at $8 \mathrm{~Hz}$, corresponding to the rhythmic cerebral activity seen in the filtered EEG of Figure 6B.

with no prior exposure to computer usage. No knowledge of the RSX-11M operating system is required. For a large number of parameters, default values are used to reduce the amount of typing needed. This system has been operated daily by EEG technologists for close to 1 year. Samples of one of the "menus" of commands and of the presentation of results are shown in Figure 8.

\section{CONCLUSION}

We have described a computer system designed to enhance the traditional recording of the EEG of epileptic patients. Patients may be monitored for several hours or several days with practically no intervention of personnel. The very long recording normally obtained in such a situation can be replaced by a series of samples containing information useful to the patient's diagnosis. The operation of the computer and the interpretation of the results have been well integrated into the routine operation of the clinical EEG laboratory. The system is also used as a quantitative research tool in clinical electroencephalography. It allows the recording on magnetic tape of a large number of epileptic seizures that can be studied later (Gotman et al., Note 1; Ives, Gotman, \& Rasmussen, Note 2). The change of interictal epileptic activity over long periods of time and its relations with blood levels of antiepileptic medication or with occurrence of seizures may also be systematically studied. Since monitoring programs are run in a standard multiprogramming environment, other people may be using the computer while monitoring is going on; the on-

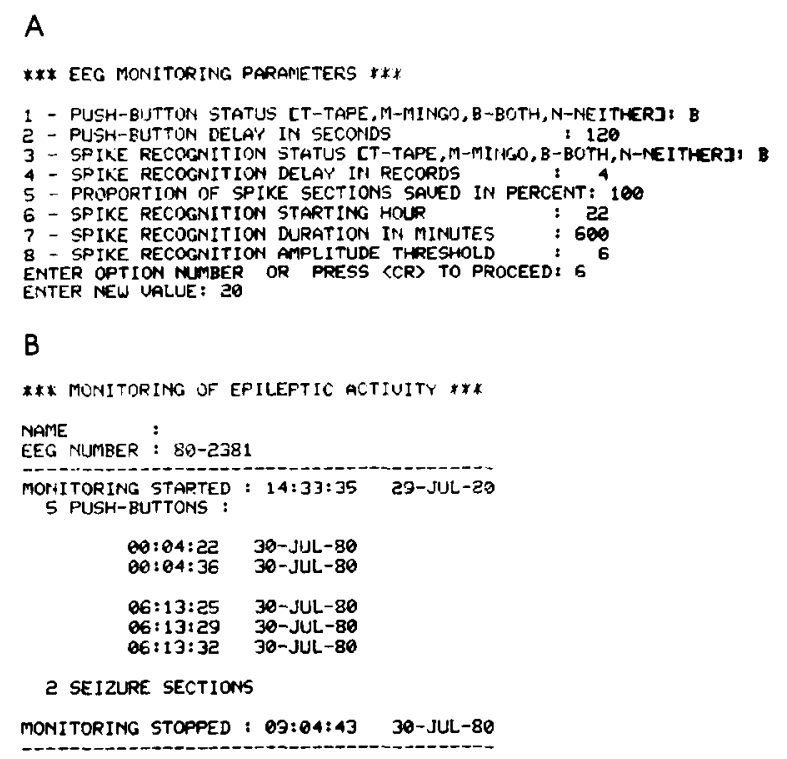

Figure 8. Examples of text appearing on the computer terminal when using the EEG monitoring system. (A) Parameters that are set prior to starting the monitoring: Default values are normally used; in this example, the user is changing the starting time of the spike recognition from $2200 \mathrm{~h}$ to $2000 \mathrm{~h}$. (B) Information on seizure activity appearing on the computer terminal when monitoring is stopped.

line detection of epileptic spikes on 16 EEG channels is, however, quite demanding of computer resources (approximately $70 \%$ of available CPU time). The present system is now being updated to allow the simultaneous 
monitoring of two patients: The EEGs of both patients will be delayed by $2 \mathrm{~min}$ to allow the recording of epileptic seizures in both, but the EEG of only one patient will be processed for automatic recognition of spikes and sharp waves.

\section{REFERENCE NOTES}

1. Gotman, J., Ives, J. R., \& Gloor, P. Frequency content of the onset of epileptic seizures. Paper presented at the annual meeting of the American EEG Society, Atlanta, 1979.

2. Ives, J. R., Gotman, J., \& Rasmussen, T. Comparison of EEG seizure onset frequency in patients with and without space occupying lesions. Paper presented at the annual meeting of the Eastern Association of Electroencephalographers, New York, 1979.

\section{REFERENCES}

BARLow, J. S. Computerized clinical electroencephalography in perspective. IEEE Transactions on Biomedical Engineering, $1979,26,377-391$.

Brazier, M. A. B. Spread of seizure discharges in epilepsy: Anatomical and electrophysiological considerations. Experimental Neurology, 1972, 36, 263-272.

Chathian, G. E., Bergamini, L., Dondey, M., Klass, D. W., Lennox-Buchthal, M., \& Petensen, I. A glossary of terms most commonly used by clinical electroencephalographers. Electroencephalography and Clinical Neurophysiology, 1974, 37, 538-548.

Cox, J. R., Nolle, F. M., \& Arthur, R. M. Digital analysis of the electroencephalogram, the blood pressure wave and the electrocardiogram. Proceedings of the IEEE, 1972, 60, 1137-1164. Gevins, A. S., Yeager, C. L., Diamond, S. L., Spire, J.-P.,
Zeitlin, G. M., \& Gevins, A. H. Automated analysis of the electrical activity of the human brain (EEG): A progress report. Proceedings of the IEEE, 1975, 63, 1382-1399.

Gotman, J., \& Gloor, P. Automatic recognition and quantification of interictal epileptic activity in the human scalp EEG. Electroencephclography and Clinical Neurophysiology, 1976, 41, 513-529.

Gotman, J., Gloor, P., \& RAy, W. F. A quantitative comparison of traditional reading of the EEG and interpretation of computerextracted features in patients with supratentorial brain lesions. Electroencephalography and Clinical Neurophysiology, 1975, 38, 623-639.

Gotman, J., Gloor, P., \& Schaul, N. Comparison of traditional reading of the EEG and automatic recognition of interictal epileptic activity. Electroencephalography and Clinical Neurophysiology, 1978, 44, 48-60.

Gotman, J., Ives, J. R., \& Gloon, P. Automatic recognition of interictal epileptic activity in prolonged EEG recordings. Electroencephalography and Clinical Neurophysiology, 1979, 46, $510-520$.

Gotman, J., Skuce, D. R., Thompson, C. J., Gloor, P., Ives, J. R., \& RAY, W. F. Clinical applications of spectral analysis and extraction of features from electroencephalogram with slow waves in adult patients. Electroencephalography and Clinical Neurophysiology, 1973, 35, 225-235.

Ives, J. R., \& GLooR, P. A long-term time-lapse video system to document the patients spontaneous clinical seizure synchronized with the EEG. Electroencephalography and Clinical Neurophysiology, 1978, 45, 412-416.

Ives, J. R., Thompson, C. J., \& Gloon, P. Seizure monitoring: A new tool in electroencephalography. Electroencephalography and Clinical Neurophysiology, 1976, 41, 422-427.

O'Donnell, R. D., Berkhout, J., \& Adey, W. R. Contamination of scalp EEG spectrum during contraction of cranio-facial muscles. Electroencephalography and Clinical Neurophysiology. $1974,37,145-151$. 